\title{
An Intelligent Tropical Cyclone Eye Fix System using Motion Field Analysis*
}

\author{
WONG Ka Yan YIP Chi Lap \\ kywongecs.hku.hk clyipdcs.hku.hk \\ Department of Computer Science, The University of Hong Kong, Hong Kong
}

\begin{abstract}
Tropical cyclones (TCS) are weather systems with vast destructive power. Accurate location of their circulation centers, or "eyes", is thus important to forecasters. However, the eye fix process is often done manually in practice. While multiple factors are considered in the process, with subjective elements in these methods, forecasters could disagree. This paper describes a TC eye fix system that uses a novel motion field structure analysis method. It can handle TCs without well-defined structure that are partially out of the image. The systems also adapts user inputs and past results to improve its accuracy. Implemented on a commodity desktop computer, the system can process about 5 images per minute, giving an average error of about 0.16 degrees in latitude/longitude on Mercator projected map for TCs that are completely inside the radar image. This is well within the relative error of about 0.3-0.4 degrees given by different TC warning centers. This TC eye fix system is useful in giving an objective TC center location in contrast to traditional manual analysis.
\end{abstract}

\section{Introduction}

Tropical cyclones (TCs) are among the most destructive weather phenomena. They form dangerous threat to life and property. Direct economic loss often amount to billions of dollars. To reduce loss, forecast centers should issue warnings early based on a TC forecast track. This requires the accurate location of the circulation center, or the "eye", of the TC. This is normally done by the analysis of remote sensing data from weather radars or satellites.

Weather radars work by sending out microwave signals to the atmosphere. The reflected signals are then preprocessed to extract the relevant slices suitable for analysis. The radar reflectivity data at $3 \mathrm{~km}$ Constant Altitude Plan Position Indicator (CAPPI) (Figure 4) and the correspond-

* The authors are thankful to the Hong Kong Observatory for provision of data and expert advice from Dr. LI Ping Wah. ing Doppler velocity data are often used for TC eye fix. The former shows the strength of echos reflected from rain, snow, ice or hail aloft, and the latter shows their radial velocities with respect to the radar.

Since a TC is a weather system with spiraling rainbands whose circulation center is the eye, the line with zero radial velocity with respect to the radar, called zero isodop, is where the TC center should lie. The radars used by the Hong Kong Observatory (HKO) [2] take six minutes to update both types of data. They cover a radius of up to $512 \mathrm{~km}$, with spatial resolution of a few hundred meters. Since TCs in the proximity of hundreds of kilometers from a city fall into the range of radars and pose the greatest threat, we focus on the eye fix process using radar images.

\section{Related Work}

In practice, TC eye fix is often done by forecasters tracing the movement of spiral rainbands or overlaying spiral templates on remote sensing images for the best match [6]. However, these methods are not completely objective. In contrast, automated methods including pattern matching and wind field analysis employ objective measures. Pattern matching TC eye fix methods require the use of a predefined TC model. For example, in our previous work [8], a TC spiral rainband is modeled by the equation $r=a e^{\theta \cot \alpha}$, where $a$ and $\alpha$ are found by transformation techniques to generate templates. Genetic algorithm is applied to speed up the search [9], and temporal information is further incorporated to improve speed and accuracy [7].

Though effective, these techniques require TCs to have the same feature set defined in the models, limiting their effectiveness on TCs that are forming, dissipating (Figure 4(k)), or partially out of radar range (Figure 4(e)). Wind field analysis, which uses both spatial and temporal information, can be used even when a well-defined TC eye is missing. Motion field built using the TREC algorithm [5] is used for eye fix [3]. It is also possible to extract critical points such as swirl or vortices from mathematical models of vector fields for TC identification [1]. However, best 

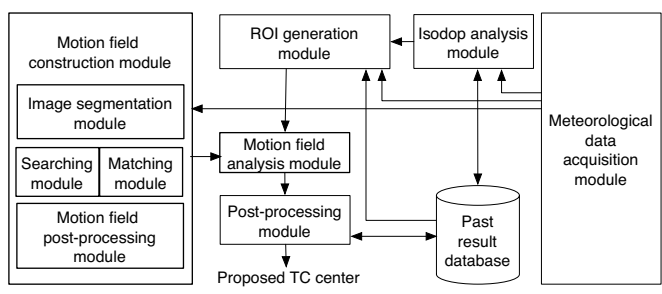

Figure 1. TC eye fix system architecture

results require well-structured motion fields and computationally expensive operations.

In this paper, we introduce an intelligent system for TC eye fix. Motion compensation techniques are used to construct motion fields. The TC centers are located objectively using a novel motion field structure analysis method. Comparison with traditional mathematical analysis of the motion field is done to evaluate its performance. The system also supports user interaction to enhance the accuracy.

\section{System Architecture}

The architecture of the TC eye fix system is shown in Figure 1. It is organized in a modular manner supporting operations including data acquisition, isodop analysis, Region Of Interest (ROI) generation, motion field construction and analysis, and post-processing. A past result database is used to store previous eye fix results and information such as ROI and zero isodop locations, for assisting the eye fix process.

The flow of the TC eye fix process is as follows. First, radar reflectivity images are obtained from the meteorological data acquisition module. Then, the images are segmented into a number of equal-sized square blocks in the image segmentation module. Next, motion estimation is applied to construct the motion field. In our system, the matching and searching modules provide a flexible interface for interchanging different distortion functions and searching algorithms. The motion field obtained is then smoothed by the post-processing module, where different filtering techniques can be applied. With the motion field in place, analysis is done in the motion field analysis module, which supports different analysis methods (see Section 5).

To reduce the search space and time needed for an eye fix exercise yet retaining a reasonable accuracy, an ROI generated by the ROI generation module is needed. It is determined using the zero isodop. The isodop analysis module is responsible for finding the zero isodop line using Doppler velocity data from the meteorological data acquisition module, and past zero isodop information from the past result database. If Doppler velocity data is unavailable, an ROI is generated by applying motion estimation techniques on the ROI of the previous image from the past result database. To allow interaction with forecasters, the ROI generation module also supports a user-specified rectangular area.

Since meteorological data may occasionally be incomplete or missing, eye fix results are often noisy. Noise reduction techniques are needed. Kalman filtering is supported in the post-processing module. The filtered TC center location gives the final output, and is stored in the past result database. These values will be adapted to the output as the filter runs.

To improve the accuracy, the system also supports user input of TC center locations. This provides a basic feedback step in which the forecaster is given the opportunity to verify the proposed TC center locations whenever necessary.

\section{Motion Field Construction}

Our TC eye fix method works on radar reflectivity images taken at intervals of several minutes (six in our experiments). Since clouds are nonrigid bodies with complex motion dynamics, to simplify the problem, it is assumed that each small region undergoes uniform rigid motion. This way, a sequence of radar images can be modeled as a video, and motion compensation techniques can be applied to construct motion fields. Each radar image is segmented into a number of equal-sized square blocks with a side length of blockSize pixels. The blocks are spaced stepSize pixels apart in both dimensions. Motion estimation is then applied onto the image sequence to derive the motion vector of each block. According to domain-specific information such as the average speed of TC, the parameters blockSize and stepSize are set to 19 and 10 respectively. A $5 \times 5$ median filter is then applied to smooth out the motion field.

\section{Motion Field Analysis}

\subsection{Circulation Analysis}

With the motion field in place, analysis is done to identify the TC eye. One way is to analyze the field mathematically by finding circulation values at potential TC center points on the image. Since strong winds generated by TCs in the northern hemisphere circulate in an anticlockwise manner around its eye, a TC center is the place having the maximum circulation. The circulation of a region is defined by the equation $\oint_{C} \vec{u} \cdot \vec{T} d s$, where $\vec{u}$ is a motion vector in the vector field, and $\vec{T}$ is a unit tangent vector along $C$. Thus, the center of a circular closed curve that follows the motion field gives the maximum circulation value. The idea is illustrated in Figure 2(a). The solid yellow curve follows the motion field. Its center has a higher circulation value than that of the pink dotted curve. By considering circles of different diameters on the motion field, a point having the maximum circulation value is considered as the TC center. 


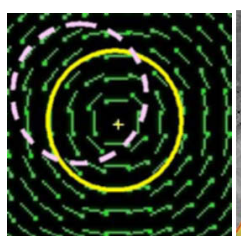

(a)Illustration of circulation analysis

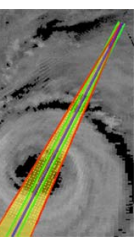

(b)ROI

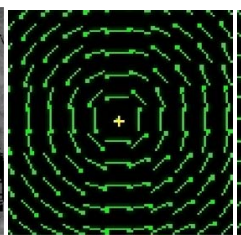

(c)Artificial circular (d)2(c) rotated by field

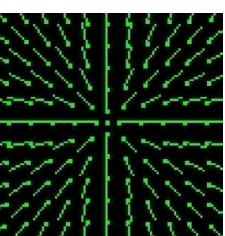

$90^{\circ} \mathrm{CCW}$

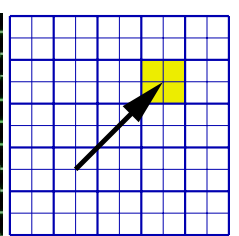

(e)Vector with \pm 0.5 (f)Sector region pixels error

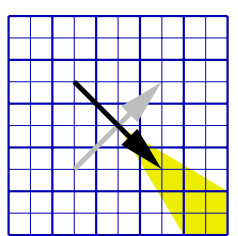

from rotated vector

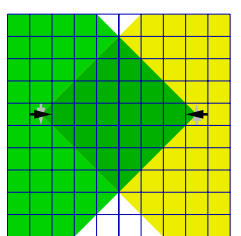

(g)Sector overlap (short vectors)

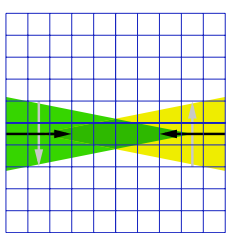

(h)Sector overlap (long vectors)

Figure 2. ROI, circulation, straight line analysis, sector analysis method and short vector problem

To increase the efficiency, the search is done only within an ROI, determined by finding the zero isodop from Doppler velocity data using a log-polar transformation. In our experiments, it is an area within $\phi=\pi / 45$ radian or $w=3 \mathrm{~km}$ from the straight zero isodop line (the shaded area in Figure 2(b)) or a user-defined rectangular area.

\subsection{Motion Field Structure Analysis}

Apart from traditional mathematical analysis, a TC eye can be found by analyzing the motion field structure. By rotating each vector of a circular motion field $90^{\circ}$ anticlockwise, a new field is formed with all vectors pointing towards the circulation center (Figures 2(c) and 2(d)). A straight line can be formed by following the direction of each rotated vector, and the circulation center is in the region with the highest number of straight lines passing through. Note that when there is combined translational and rotational motion, the motion field would be asymmetric, causing an apparent shift of circulation center. Thus, the magnitude of rotated vectors are ignored in this straight line analysis method to minimize the translation effect on the center region.

\subsection{Variations}

Since motion estimation algorithms often give motion vectors with integer pixel coordinates, there is a maximum error of \pm 0.5 pixels on each dimension of a vector. Taking this error into consideration, a vector in the field can be seen to represent any vector whose end point fall into the shaded region of Figure 2(e). Therefore, in straight line analysis, a straight line formed from a rotated vector is turned to a sector, as shown in Figure 2(f). This sector region bounds the locations of all possible straight lines formed by the set of vectors derived from the motion vector with integer coordinates. The circulation center of this sector analysis method is in the area covered by the highest number of sector regions.

Since a sector region derived from an integer vector is used, error introduced by vectors with short magnitude can be relatively large. Figures $2(\mathrm{~g})$ and $2(\mathrm{~h})$ show the problem caused by short vectors. The sector region derived from short vectors covers a larger area compared with the one formed by long vectors. Some insignificant areas are therefore included during analysis. To reduce this effect, we can filter out all vectors having a magnitude smaller than shortLength $\mathrm{km}$. With reference to the average wind speed caused by a typical TC, shortLength is set to 2.1 in our experiments.

\section{Result Post-processing}

To smooth out the noise caused by image defects and motion prediction errors, the (lon, lat) TC center locations found are fed to a Kalman filter. Historical TC data, such as average TC speed, are used to determine Kalman filter parameters such as system noise variance. Latitude and longitude values are separately smoothed, with the assumption that they are statistically independent. The Kalman filtered TC center location gives the output of the whole system.

\section{Experimental Design}

Applying the variations to motion field analysis methods introduced in Section 5 gives five methods for TC eye fix: circulation analysis, straight line analysis, sector analysis, straight line analysis with filtering, and sector analysis with filtering. To evaluate their efficiency and effectiveness, a Java-based system is built. Sequences of $3 \mathrm{~km}$ CAPPI radar reflectivity images with a range of $256 \mathrm{~km}$ captured every 6 minutes, along with their Doppler velocity counterparts, were used for testing. The experimental data include Typhoon Yutu, HKT 2001-07-24 20:00 to 2001-07-25 19:54 (240 images), Typhoon Imbudo, HKT 2003-07-23 13:00 to 2003-07-24 13:54 (250 images), and STS Maria, HKT 2000-08-31 22:00 to 2000-09-01 14:54 (180 images).

The efficiency of the algorithm is evaluated using the average number of images the system can process in a minute on a desktop computer. It has a $2 \mathrm{GHz}$ Pentium 4 processor and 1G RAM and runs Red Hat Linux 9.0 with kernel version 2.4.20-8. The effectiveness is evaluated by finding the average Euclidean distance between the algorithm proposed center and the corresponding interpolated best track location from HKO. Best tracks are the hourly TC locations 
determined after the event by a TC warning center using all available data. According to domain-specific information such as typical TC strength, the parameters blockSize and stepSize are set to 19 and 10 pixels respectively. The effect of motion field density is also investigated by varying blockSize to 16, and stepSize to 5 and 14.

\section{Results and Evaluation}

\subsection{Efficiency}

Due to space limitations, only more representative experimental results are presented. Using the chosen parameters, the system processes around 5 images a minute on average. Figure 3(c) shows the effect of blockSize and stepSize on the processing rate using STS Maria in experiments. In general, all methods run slower as the density of motion field increases (i.e., blockSize and stepSize decrease). This is because more time is needed in block matching and derivation of motion vectors. An investigation on the efficiency of different methods shows that straight line analysis and sector analysis run faster than circulation, as less mathematical computations are involved. The system runs even faster while straight line or sector analysis methods with short vectors filtering are used, as insignificant vectors are neglected in computation and speed up the search.

\subsection{Effectiveness}

Using the chosen parameters, for TCs with a welldefined center inside radar range, such as Typhoon Yutu, all methods give an average error within 0.16 degrees in latitude/longitude on the Mercator projected map. This is well within the relative error of about 0.3-0.4 degrees given by different TC warning centers [4]. The best track, along with the tracks proposed by different methods, are shown in Figure 3(a). At the initial stage of Yutu, it is still a relatively small weather system (Figures 4(a) and 4(b)). Only a few motion vectors can be derived for analysis, giving answers with a relatively large deviation from the best track. Yet, in general, for the whole sequence, all the proposed centers are close to the best track. Figures 4(c) and 4(d) show one of the eye fix result using sector analysis with filtering.

To see how effective the methods are in handling TCs that are out of radar range, data of Typhoon Imbudo, whose eye is outside the radar range most of the time, is used. Circulation gives an average error of 0.70 degrees, while straight line and sector analysis methods with filtering give an average error of 0.42 and 0.47 degrees respectively. An inspection of radar images shows that circulation considered the outermost rainbands as the circular curve having the maximum circulation instead of the eye wall, hence giving answers far away from the correct ones. Since the TC eye is out of radar range (Figures 4(e) and 4(f)), vectors around the eye are counted as zero vectors, and are ignored in straight line and sector analysis methods with filtering. The remaining vectors provide a spiral structure, which enable the methods to estimate the TC center. This estimation, though not very accurate, help forecasters to predict the direction and trend of TC movement. Straight line and sector analysis methods with filtering also give answers close to the best track before Imbudo enters radar range, as illustrated in Figure 3(b). Such relatively small error allows Kalman filter to converge to the correct answers rapidly when Imbudo enters range of radar (Figures 4(g) and 4(h)).

The comparison on accuracy using STS Maria, which is a TC dissipated within the radar range, is shown in Figure 3(d). Though within radar range, in general, the proposed methods give an average error within 0.35 degrees, which is relatively large while compared with Typhoon Yutu. This is because at the late stage of Maria, it is dissipated and the eye is vanished (Figures 4(k) and 4(l)). Among all the methods, circulation gives the largest error which is affected by the weak circulation structure at the late stage. In contrast, straight line analysis and sector analysis methods, which give smaller errors, do not require very well-structured circular fields. Figures 4(i) and 4(j) show one of the eye fix results of STS Maria before its dissipation using sector analysis with filtering.

The effect of blockSize and stepSize on accuracy using STS Maria is shown in Figure 3(d). It can be seen that denser vector fields do not have strong effects on accuracy. This insensitivity of error values to blockSize and stepSize is an advantage as the parameters can be chosen to maximize the processing speed without affecting the error much.

\section{Summary and Future Work}

We have introduced a system that makes use of a novel motion field analysis method to solve the TC eye fix problem. A sequence of radar images is modeled as a video, and motion compensated prediction is applied to construct motion vector fields for analysis. Using three TC sequences with different properties, four new methods for analyzing the motion field structures, along with the mathematical analysis of circulation values, are compared. These methods are straight line analysis, sector analysis, straight line analysis with filtering, and sector analysis with filtering. It was found that they can process about 5 images per minute when implemented on a commodity desktop computer. The average error is about 0.16 degrees in latitude/longitude on the Mercator projected map for TCs that are completely inside the image, well within the relative error of about 0.3 0.4 degrees given by different TC warning centers. The accuracy is found to be relatively insensitive to the density of the motion field. The problem that plagues traditional vec- 


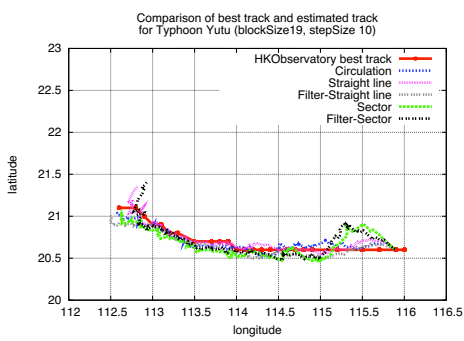

(a)Estimated and best tracks: Yutu

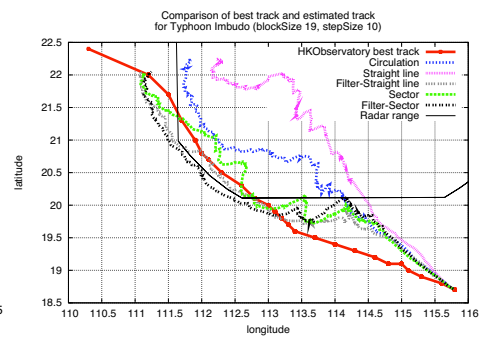

(b)Estimated and best tracks: Imbudo

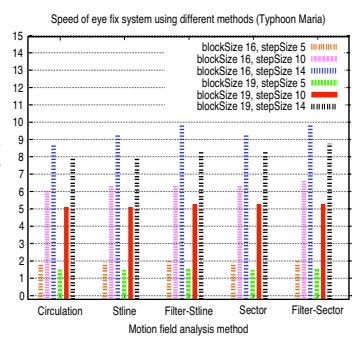

(c)Field density: Speed

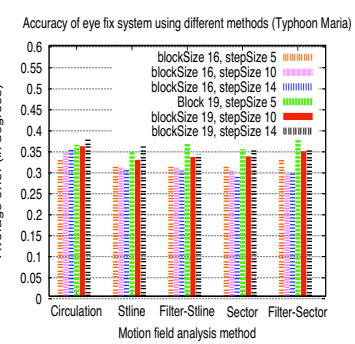

(d)Field density: Accuracy

Figure 3. Tracks comparison and effect of motion field density (blockSize and stepSize)

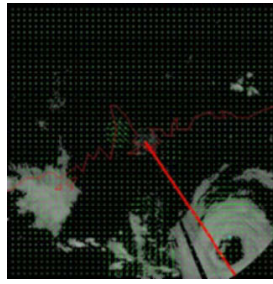

(a)Typhoon Yutu, 2001-07-24 11:20

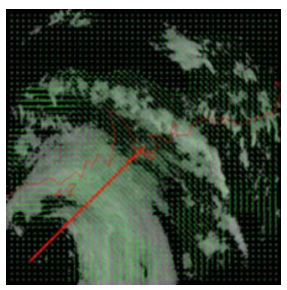

(g)Imbudo, eye entered (h)Figure 4(g): Zoomed range, 2003-07-24 04:48 in

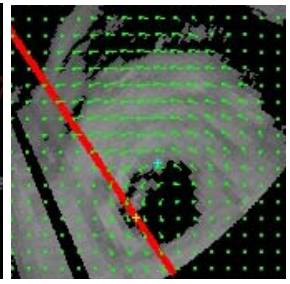

(b)Figure 4(a): Zoomed in

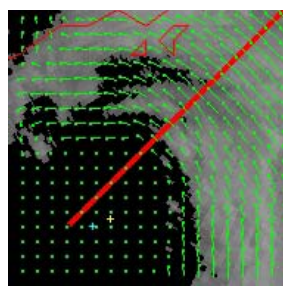
in

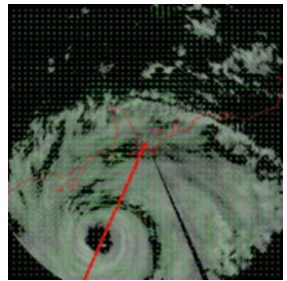

(c)Yutu, well defined eye, 2001-07-25 13:18

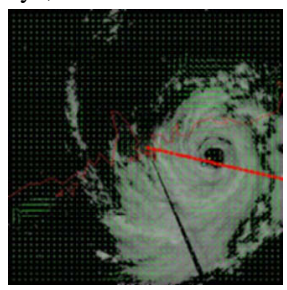

(i)Maria, well defined eye, 2000-09-01 00:36

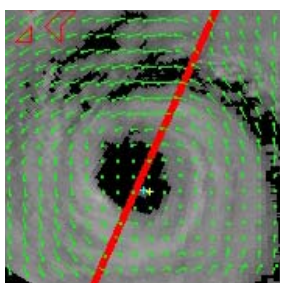

(d)Figure 4(c): Zoomed in

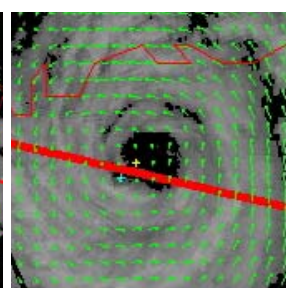

(j)Figure 4(i): Zoomed in(k)Maria, dissipated, 2000-09-01 09:48

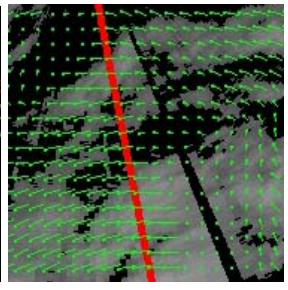

(f)Figure 4(e): Zoomed range, 2003-07-23 16:24 in
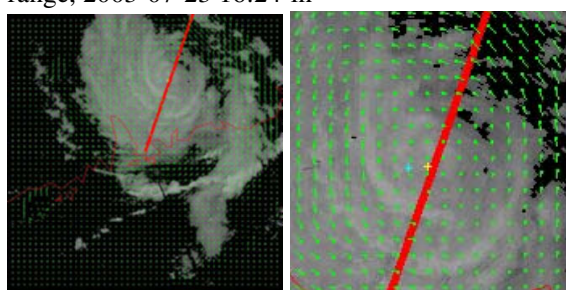

(l)Figure 4(k): Zoomed in

Figure 4. Results of TC eye fix using sector analysis with filtering method. (Red line: zero isodop; Yellow "+": Proposed TC center; Cyan "+": Kalman filtered TC center)

tor field analysis methods, namely the apparent shift of circulation center caused by asymmetric motion fields due to combined translational and rotational motions, is also minimized in the proposed methods. The introduced methods are particularly useful for TCs that are dissipating or outside the radar range, since the estimation of eye locations gives clues for forecasters to predict and forecast weather. The TC eye fix system is useful in giving an objective TC center location in contrast to traditional manual analysis.

\section{References}

[1] T. Corpetti, E. Mémin, and P. Pérez. Extraction of singular points from dense motion fields: An analytic approach. $J$. Mathematical Imaging and Vision, 19:175-198, 2003.

[2] Hong Kong Observatory. http://www.hko.gov.hk/. Web page, 2005.

[3] E. S. T. Lai. TREC application in tropical cyclone observation. In ESCAP/WMO Typhoon Committee Annual Review, pages 135-139, 1998.
[4] C. Y. Lam. Operational tropical cyclone forecasting from the perspective of a small weather service. In Proc. ICSU/WMO Sym. Tropical Cyclone Disasters, pages 530-541, Oct. 1992.

[5] R. E. Rinehart. Internal storm motions from a single nonDoppler weather radar. Technical Report TN-146+STR, National Center for Atmospheric Research, 1979. 262 pages.

[6] M. V. Sivaramakrishnan and M. Selvam. On the use of the spiral overlay technique for estimating the center positions of tropical cyclones from satellite photographs taken over the Indian region. In Proc. 12th Conference on Radar Meteorology, pages 440-446, 1966.

[7] K. Y. Wong and C. L. Yip. Tropical cyclone eye fix using genetic algorithm with temporal information. In Proc. 9th KES, LNAI-3681, pages 854-860, Australia, Sept. 2005.

[8] K. Y. Wong, C. L. Yip, P. W. Li, and W. W. Tsang. Automatic template matching method for tropical cyclone eye fix. In Proc. 17th ICPR, volume 3, pages 650-653, 2004.

[9] C. L. Yip and K. Y. Wong. Efficient and effective tropical cyclone eye fix using genetic algorithms. In Proc. 8th KES, LNAI-3213, pages 654-660, New Zealand, Sept. 2004. 